\title{
Unified Green's function retrieval by cross-correlation; connection with energy principles
}

\author{
Roel Snieder, ${ }^{1, *}$ Kees Wapenaar, ${ }^{2}$ and Ulrich Wegler ${ }^{3}$ \\ ${ }^{1}$ Center for Wave Phenomena and Department of Geophysics, Colorado School of Mines, Golden, Colorado 80401, USA \\ ${ }^{2}$ Department of Geotechnology, Delft University of Technology, 2600 GA Delft, The Netherlands \\ ${ }^{3}$ Institut für Geophysik und Geologie, Universität Leipzig, Leipzig, Germany
}

(Received 13 October 2006; published 2 March 2007)

\begin{abstract}
It has been shown theoretically and observationally that the Green's function for acoustic and elastic waves can be retrieved by cross-correlating fluctuations recorded at two locations. We extend the concept of the extraction of the Green's function to a wide class of scalar linear systems. For systems that are not invariant under time reversal, the fluctuations must be excited by volume sources in order to satisfy the energy balance (equipartitioning) that is needed to extract the Green's function. The general theory for retrieving the Green's function is illustrated with examples that include the diffusion equation, Schrödinger's equation, a vibrating string, the acoustic wave equation, a vibrating beam, and the advection equation. Examples are also shown of situations where the Green's function cannot be extracted from ambient fluctuations. The general theory opens up new applications for the extraction of the Green's function from field correlations that include flow in porous media, quantum mechanics, and the extraction of the response of mechanical structures such as bridges.
\end{abstract}

DOI: 10.1103/PhysRevE.75.036103 PACS number(s): 43.20.+g, 46.40.Cd, 03.65.- w, 46.65.+g

\section{INTRODUCTION}

The extraction of the Green's function from ambient fluctuations for acoustic and elastic waves has recently received much attention: see recent tutorials and reviews [1,2], and the special supplement on seismic interferometry in Geophysics [3]. Derivations of this principle have been based on normal modes [4], on representation theorems $[5,6]$, on the superposition of incoming plane waves [7-9], on timereversal invariance $[10,11]$, and on the principle of stationary phase [12-15]. The extraction of the Green's function has been applied to ultrasound [16-18], to crustal seismology [19-23], to exploration seismology [24-26], to helioseismology [27-29], to structural engineering [30-32], to ocean acoustics $[33,14,34]$, to earthquake data recorded in a borehole [35], and to monitoring of volcanoes and fault zones $[36,37]$.

Wapenaar et al. [38] derived the extraction of the Green's function for systems of coupled first-order differential equations that describe general linear systems that include acoustic and elastic waves, the Maxwell's equations, and diffusive systems. The general applicability of the extraction of the Green's function is reminiscent to the fluctuation-dissipation theorem, e.g., Refs. [39-41], which states that the response of a linear system in thermodynamic equilibrium to an external force is related to the fluctuations in the system. The application of the fluctuation dissipation theorem to macroscopic systems such as the Earth's crust or ocean is, however, not trivial. The energy of macroscopic systems is large compared to the thermal energy; hence these systems are, in general, not in thermodynamic equilibrium. The extraction of the Green's function for acoustic and electromagnetic waves was derived earlier for stationary random media [42-44]. These treatments rely on an ensemble average, and therefore give the Green's function of the mean field only. In this work

*Electronic address: rsnieder@mines.edu we average neither over thermal fluctuations, nor over an ensemble, but use an averaging over sources instead.

In this work we explore the general formulation of the extraction of the Green's function for linear scalar systems and explore the requirements that such a system must satisfy in order to retrieve the Green's function from fluctuations. Section II illustrates the central role of the concept of equipartitioning in the extraction of the Green's function. We introduce linear systems with symmetric spatial differential operators in Sec. III, and derive in Sec. IV a general theory for the extraction of the Green's function from fluctuations for such systems. Several examples are shown that are either of a didactic nature, or because they provide new applications. This general formalism is applied to systems that are invariant under time reversal (Sec. V), to the diffusion equation (Sec. VI), to a string with either an open end or fixed ends (Secs. VII and VIII), to acoustic waves (Sec. X), to Schrödinger's equation (Sec. XI), and to a vibrating beam (Sec. XII). We extend the general theory to antisymmetric differential operators in Sec. XIII, and apply this in Sec. XIV to the one-dimensional advection equation. In Sec. IX we show that the Green's function cannot always be retrieved by cross-correlation, and relate this to the lack of equipartitioning. We explain in Sec. XV why energy transport plays such a central role in the retrieval of the Green's functions from ambient fluctuations. In the Appendix we show that the requirement of equipartitioning is stronger than the condition that the net energy current vanishes.

\section{A HEURISTIC EXPLANATION OF THE ROLE OF EQUIPARTITIONING}

The concept of equipartitioning plays a central role in the retrieval of the Green's function. The word equipartitioning is often used to mean that all modes $[2,45]$, or degrees of freedom [46], of the system are excited with equal energy. It has also been used to indicate that the energy current is equal in all directions [18]. We use the latter definition of equipar- 


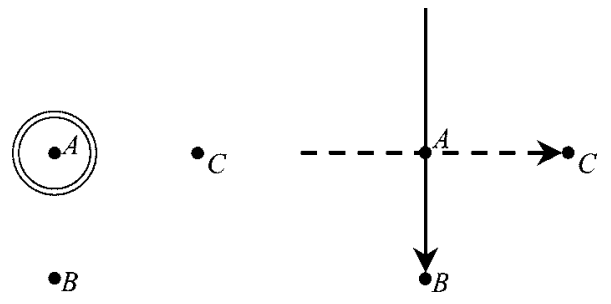

FIG. 1. Left panel: a point source in a homogeneous acoustic medium at point $A$ that emits equal amounts of energy toward points $B$ and $C$. Right panel: equal energy transport along the solid and dashed arrows is needed to retrieve the Green's function for the propagation from the source point $A$ to the points $B$ and $C$.

titioning. Figure 1 serves to heuristically illustrate the central role of equipartitioning. In this example we consider acoustic waves in a homogeneous medium. A pressure source at location $A$ excites acoustic waves. As shown in the left panel, these waves propagate with equal amplitude to receivers at points $B$ and $C$.

Next, we consider the retrieval of the Green's function from cross-correlation. In the right panel of Fig. 1 there is no physical source at point $A$, and incoming waves turn into outgoing waves from point $A$ as they pass through that location. Because of the absence of a physical source in the right panel of Fig. 1, the term virtual source has been used $[24,25]$. Since the outgoing waves for the physical source in the left panel have the same amplitude for all propagation directions, the same must be true for the virtual source in the right panel. The outgoing wave at point $A$ in the right panel has the same energy as the incoming wave, because the waves simply move through point $A$. The equivalence of the real source in the left panel, and the virtual source in the right panel, implies that the incoming waves in the right panel must have the same energy for all propagation directions. In other words: the waves must be equipartitioned. For the sake of argument we used a homogeneous acoustic medium. This is, however, not essential. A scalar source in an inhomogeneous medium also radiates waves isotropically [47].

As another case consider the situation that point $B$ is a diffractor. The direct wave that travels from $A$ to $C$ should be excited by the virtual source with the same strength as that of the diffracted wave that travels from $A$ through the diffractor $B$ to point $C$. In order to retrieve the correct amplitude ratio of the direct and diffracted waves from cross-correlation, the energy currents along the solid and dashed arrows in the right panel of Fig. 1 must be identical.

For vector equations, such as for elastic waves, the energy is usually not radiated isotropically. For example, a point force in an elastic medium radiates energy with a dipole pattern [48]. For such a vector problem, one retrieves the Green's function by cross-correlating the displacement fields recorded at two receivers [5]. The projection of the displacement field onto the component used for the cross-correlation, gives the same dependence on the direction of propagation as does the radiation pattern of a point force. Therefore, one also needs the energy current to be independent of direction for such a vector problem.
In general, the extraction of the Green's function by crosscorrelation gives the superposition of the causal Green's function and its time-reversed version (usually called the acausal Green's function.) In observational studies the causal Green's function as estimated by cross-correlation, and its acausal counterpart, often have different amplitudes. This asymmetry has been linked to the lack of equipartitioning (e.g., Refs. $[18,49])$. We give a more quantitative discussion of equipartitioning in Sec. XV.

The requirement that the energy current is independent of direction implies that the net energy current vanishes. The net energy current vanishes when the energy current for every pair of opposing directions vanishes, but the energy current can still vary with direction. We show in the Appendix explicitly that a vanishing net energy current does not necessarily imply equipartitioning.

\section{A GENERAL DYNAMIC SYSTEM WITH A SYMMETRIC OPERATOR}

Consider a scalar field $u$ that is governed by the equation

$$
\begin{aligned}
& \left(a_{N}(\mathbf{r}, t) * \frac{\partial^{N}}{\partial t^{N}}+\cdots+a_{2}(\mathbf{r}, t) * \frac{\partial^{2}}{\partial t^{2}}+a_{1}(\mathbf{r}, t) * \frac{\partial}{\partial t}\right) u(\mathbf{r}, t) \\
& \quad=H(\mathbf{r}, t) * u(\mathbf{r}, t)+q(\mathbf{r}, t) .
\end{aligned}
$$

In this expression the asterisk denotes temporal convolution, $q(\mathbf{r}, t)$ is the forcing, and $H$ is a symmetric operator with properties that are defined in Eq. (3). Later we provide examples of physical systems that are described by Eq. (1). For Schrödinger's equation, the wave function is complex in the time domain, hence $u(\mathbf{r}, t)$, and the time-domain Green's function, may be complex. In this work we analyze this system in the frequency domain, using the Fourier convention, $h(t)=\int h(\omega) \exp (-i \omega t) d \omega$. With this convention, expression (1) corresponds, in the frequency domain, to

$$
\sum_{n=1}^{N} a_{n}(\mathbf{r}, \omega)(-i \omega)^{n} u(\mathbf{r}, \omega)=H(\mathbf{r}, \omega) u(\mathbf{r}, \omega)+q(\mathbf{r}, \omega)
$$

Henceforth we suppress the frequency dependence of these quantities. The operator $H(\mathbf{r}, \omega)$ and the coefficients $a_{n}(\mathbf{r}, \omega)$ are not necessarily real.

Symmetry of $H$ means that for any two functions $f$ and $g$

$$
\int_{V_{\mathrm{tot}}} f(H g) d V=\int_{V_{\mathrm{tot}}}(H f) g d V
$$

where the integration is over the total volume $V_{\text {tot }}$ over which the system is defined. For example, in seismology the total volume could be the solid earth, which is bounded by a stress-free surface. Because of property (3), the system satisfies reciprocity. To show this we derive a representation theorem of the convolution type by considering expression (2) for two states that we label with the subscripts $A$ and $B$, by evaluating $(2)_{A} u_{B}-u_{A}(2)_{B}$. In this notation $(2)_{A}$ denotes expression (2) for state $A$. This subtraction gives 


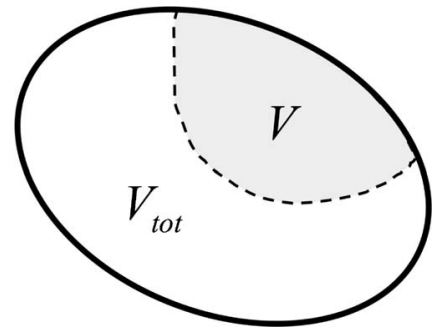

FIG. 2. Definition of the total volume $V_{\text {tot }}$ (bounded by the solid line), and the subvolume $V$ defined by the shaded region.

$$
u_{A}\left(H u_{B}\right)-\left(H u_{A}\right) u_{B}-q_{A} u_{B}+q_{B} u_{A}=0 .
$$

Integrating this equation over the total volume $V_{\text {tot }}$, and using Eq. (3) gives

$$
\int_{V_{\mathrm{tot}}} q_{A} u_{B} d V=\int_{V_{\mathrm{tot}}} q_{B} u_{A} d V
$$

Consider an impulsive forcing at location $\mathbf{r}_{A}$ for state $A$ and at location $\mathbf{r}_{B}$ for state $B$ :

$$
q_{A, B}(\mathbf{r})=\delta\left(\mathbf{r}-\mathbf{r}_{A, B}\right) .
$$

The response to such a forcing is, by definition, the Green's function

$$
u_{A, B}(\mathbf{r})=G\left(\mathbf{r}, \mathbf{r}_{A, B}\right) .
$$

Inserting these expressions into Eq. (5) gives

$$
G\left(\mathbf{r}_{A}, \mathbf{r}_{B}\right)=G\left(\mathbf{r}_{B}, \mathbf{r}_{A}\right),
$$

which states that reciprocity is satisfied.

As stated in expression (3), the operator is symmetric when integrated over the total volume $V_{\text {tot }}$. In this work we limit the integration in several examples to a subvolume $V$ of the total volume $V_{\text {tot }}$, see Fig. 2. For example, in seismology the subvolume may be the region that is investigated in a seismic survey. Its boundary $\partial V$ is not necessarily a physical boundary where homogeneous boundary conditions apply. For integration over this sub-volume the operator $H$ is not necessarily symmetric, and we define the bilinear form $L(f, g)$ by

$$
\int_{V}[f(H g)-(H f) g] d V \equiv \oint_{\partial V} L(f, g) d S .
$$

Examples of the bilinear form $L$ are shown in later sections. From definition (9), $L$ is antisymmetric:

$$
L(f, g)=-L(g, f) .
$$

Integrating expression (4) over the volume $V$, and using definition (9), gives

$$
\oint_{\partial V} L\left(u_{A}, u_{B}\right) d S+\int_{V}\left(q_{B} u_{A}-q_{A} u_{B}\right) d V=0 .
$$

Consider a state $B$ that is excited by an impulsive excitation at location $\mathbf{r}_{0}$, i.e., $q_{B}(\mathbf{r})=\delta\left(\mathbf{r}-\mathbf{r}_{0}\right)$; hence $u_{B}$ is given by the
Green's function $u_{B}(\mathbf{r})=G\left(\mathbf{r}, \mathbf{r}_{0}\right)$. Dropping the subscript $A$, expression (11) is then given by

$$
\oint_{\partial V} L\left[u(\mathbf{r}), G\left(\mathbf{r}, \mathbf{r}_{0}\right)\right] d S-\int_{V} G\left(\mathbf{r}, \mathbf{r}_{0}\right) q(\mathbf{r}) d V+u\left(\mathbf{r}_{0}\right)=0 .
$$

Using reciprocity, expression (8), this gives the representation theorem that relates the field to the excitation and its values on the boundary

$$
u\left(\mathbf{r}_{0}\right)=\int_{V} G\left(\mathbf{r}_{0}, \mathbf{r}\right) q(\mathbf{r}) d V-\oint_{\partial V} L\left[u(\mathbf{r}), G\left(\mathbf{r}_{0}, \mathbf{r}\right)\right] d S .
$$

\section{GENERAL EXPRESSION FOR THE EXTRACTION OF THE GREEN'S FUNCTION}

In this section we derive a general expression for the extraction of the Green's function using a representation theorem of the correlation type. Following Fokkema and van den Berg $[50,51]$ we evaluate $(2)_{A} u_{B}^{*}-u_{A}^{*}(2)_{B}$, where $(2)_{B}^{*}$ denotes, for example, the complex conjugate of expression (2) for state $B$. Integrating the result over the volume $V$ gives

$$
\begin{aligned}
& \sum_{n=1}^{N} \int_{V}\left[(-i \omega)^{n} a_{n}-(i \omega)^{n} a_{n}^{*}\right] u_{A} u_{B}^{*} d V \\
& \quad=\int_{V}\left[u_{B}^{*}\left(H u_{A}\right)-\left(H^{*} u_{B}^{*}\right) u_{A}\right] d V+\int_{V}\left(q_{A} u_{B}^{*}-q_{B}^{*} u_{A}\right) d V .
\end{aligned}
$$

For even values of $n, a_{n}(-i \omega)^{n}-a_{n}^{*}(i \omega)^{n}=2 i \operatorname{Im}\left(a_{n}\right)(i \omega)^{n}$, while for odd values of $n, a_{n}(-i \omega)^{n}-a_{n}^{*}(i \omega)^{n}=-2 \operatorname{Re}\left(a_{n}\right)$ $\times(i \omega)^{n}$. Writing $H^{*}=H+\left(H^{*}-H\right)=H-2 i \operatorname{Im}(H)$, and using definition (9) gives

$$
\begin{aligned}
& -2 \sum_{n \text { odd }} \int_{V}(i \omega)^{n} \operatorname{Re}\left(a_{n}\right) u_{A} u_{B}^{*} d V \\
& +2 i \sum_{n \text { even }} \int_{V}(i \omega)^{n} \operatorname{Im}\left(a_{n}\right) u_{A} u_{B}^{*} d V \\
& =2 i \int_{V} u_{A} \operatorname{Im}(H) u_{B}^{*} d V+\oint_{\partial V} L\left(u_{B}^{*}, u_{A}\right) d S \\
& +\int_{V}\left(q_{A} u_{B}^{*}-q_{B}^{*} u_{A}\right) d V .
\end{aligned}
$$

The general expression for the extraction of the Green's function follows by choosing expression (6) for the excitations $q_{A, B}$. According to expression (7), the fields $u_{A, B}$ are then given by the Green functions. Using reciprocity [Eq. (8)], we can write the result as 


$$
\begin{aligned}
G\left(\mathbf{r}_{A}, \mathbf{r}_{B}\right)-G^{*}\left(\mathbf{r}_{A}, \mathbf{r}_{B}\right)= & 2 \sum_{n \text { odd }}(i \omega)^{n} \int_{V} \operatorname{Re}\left[a_{n}(\mathbf{r})\right] G\left(\mathbf{r}_{A}, \mathbf{r}\right) G^{*}\left(\mathbf{r}_{B}, \mathbf{r}\right) d V-2 i \sum_{n \text { even }}(i \omega)^{n} \int_{V} \operatorname{Im}\left[a_{n}(\mathbf{r})\right] G\left(\mathbf{r}_{A}, \mathbf{r}\right) G^{*}\left(\mathbf{r}_{B}, \mathbf{r}\right) d V \\
& +\oint_{\partial V} L\left[G^{*}\left(\mathbf{r}_{B}, \mathbf{r}\right), G\left(\mathbf{r}_{A}, \mathbf{r}\right)\right] d S+2 i \int_{V} G\left(\mathbf{r}_{A}, \mathbf{r}\right) \operatorname{Im}(H) G^{*}\left(\mathbf{r}_{B}, \mathbf{r}\right) d V .
\end{aligned}
$$

In the following sections we give examples of how this expression can be used to extract the Green's function from the correlation of fields. The left-hand side is the difference of the Green's function and its complex conjugate. Since complex conjugation in the frequency domain corresponds in the time domain to time-reversal, the left-hand side corresponds in the time domain to $G\left(\mathbf{r}_{A}, \mathbf{r}_{B}, t\right)-G^{*}\left(\mathbf{r}_{A}, \mathbf{r}_{B},-t\right)$, the difference of the causal Green's function and the complex conjugate of the acausal Green's function. In many applications, such as acoustics or diffusion, the field is real in the time domain. In quantum mechanics, the wave function, and the associated time domain Green's function, is complex. For this reason we retain the complex conjugation of the time domain acausal Green's function.

The minus sign in the left-hand side of Eq. (16) is a matter of convention only. Multiplying expression (16) with $-i \omega$, and defining

$$
G^{(v)} \equiv-i \omega G
$$

gives

$$
\begin{aligned}
G^{(v)}\left(\mathbf{r}_{A}, \mathbf{r}_{B}\right)+G^{(v)^{*}}\left(\mathbf{r}_{A}, \mathbf{r}_{B}\right)= & 2 \sum_{n \text { odd }}(i \omega)^{n-1} \int_{V} \operatorname{Re}\left[a_{n}(\mathbf{r})\right] G^{(v)}\left(\mathbf{r}_{A}, \mathbf{r}\right) G^{(v)^{*}}\left(\mathbf{r}_{B}, \mathbf{r}\right) d V-2 i \sum_{n \text { even }}(i \omega)^{n-1} \int_{V} \operatorname{Im}\left[a_{n}(\mathbf{r})\right] G^{(v)}\left(\mathbf{r}_{A}, \mathbf{r}\right) \\
& \times G^{(v) *}\left(\mathbf{r}_{B}, \mathbf{r}\right) d V+\frac{1}{i \omega} \oint_{\partial V} L\left[G^{(v)^{*}}\left(\mathbf{r}_{B}, \mathbf{r}\right), G^{(v)}\left(\mathbf{r}_{A}, \mathbf{r}\right)\right] d S+\frac{2}{\omega} \int_{V} G^{(v)}\left(\mathbf{r}_{A}, \mathbf{r}\right) \operatorname{Im}(H) G^{(v) *}\left(\mathbf{r}_{B}, \mathbf{r}\right) d V
\end{aligned}
$$

This equation is equivalent to expression (16), but has a plus sign on the left-hand side, as in several other derivations, e.g., Refs. $[52,12,6]$. When $G$ denotes, for example, the displacement Green's function, $G^{(v)}$ corresponds to the Green's function for the velocity. The sign in the left-hand side of expressions (16) and (18) is thus defined by the choice of the Green's function that one uses. Note that the right-hand sides of these equations differ by a factor $1 / i \omega$. Since, with the employed Fourier convention, $-i \omega$ corresponds, to differentiation in the time domain, expressions (16) and (18) differ, in the time domain, by an additional time derivative.

\section{EXAMPLE: INVARIANT SYSTEMS UNDER TIMEREVERSAL}

Consider systems that are invariant under time reversal. This invariance has explicitly been used in some derivations for extraction of the Green's function [10,11]. An example of systems that are invariant under time reversal is, for example, the acoustic wave equation without attenuation:

$$
\frac{1}{\rho c^{2}} \frac{\partial^{2} u}{\partial t^{2}}-\nabla \cdot\left(\frac{1}{\rho} \nabla u\right)=q
$$

with $\rho$ the mass density and $c$ the speed of sound. In the notation of expression (1), $a_{2}=1 / \rho c^{2}$, and $H=\boldsymbol{\nabla} \cdot \rho^{-1} \boldsymbol{\nabla}$. For this problem only $a_{2}$ is nonzero, and both $a_{2}$ and $H$ are real. Another example is Schrödinger's equation [53]

$$
i \hbar \frac{\partial \psi}{\partial t}=-\frac{\hbar^{2}}{2 m} \nabla^{2} \psi+V \psi
$$

which is invariant under time reversal and complex conjugation. Since the complex conjugation does not change expectation values [53], the complex conjugate wave function corresponds to the same physical state. In this case only $a_{1}$ is nonzero, $H$ is real, and $a_{1}=i \hbar$ is purely imaginary.

For these examples the first two terms in the right-hand side of expression (16) vanish. Since time-reversal corresponds, in the frequency domain, to complex conjugation, time reversal invariance of Eq. (2) implies that $a_{n}(-i \omega)^{n}$ is real. For even $n$ this means that $a_{n}$ is real, while for odd $n, a_{n}$ is imaginary. Under these conditions the first two terms in the right-hand side of Eq. (16) are equal to zero, while the condition that $H$ is real implies that the last term vanishes as well. In this case, expression (16) reduces to

$$
G\left(\mathbf{r}_{A}, \mathbf{r}_{B}\right)-G^{*}\left(\mathbf{r}_{A}, \mathbf{r}_{B}\right)=\oint_{\partial V} L\left[G^{*}\left(\mathbf{r}_{B}, \mathbf{r}\right), G\left(\mathbf{r}_{A}, \mathbf{r}\right)\right] d S .
$$

This expression contains a surface integral, but no volume integral. In later sections we show that this allows for the 
extraction of the Green's function by cross-correlation of fields that are excited by sources on the surface $\partial V$ only. The general expression (16) contains both a surface integral and volume integrals. The presence of volume integrals indicates that for systems that are not invariant under time reversal, one needs sources throughout the volume to extract the Green's function. We analyze the acoustic wave equation and Schrödinger's equation in more detail in Secs. X and XI.

\section{EXAMPLE: THE DIFFUSION EQUATION}

The general expression (16) is also valid for systems that are not invariant under time reversal. As an example of such a system consider the diffusion equation

$$
\frac{\partial u(\mathbf{r}, t)}{\partial t}=\boldsymbol{\nabla} \cdot[D(\mathbf{r}) \boldsymbol{\nabla} u(\mathbf{r}, t)]+q(\mathbf{r}, t),
$$

where the diffusion constant $D(\mathbf{r})$ can vary with location. In the notation of expression (1), $a_{1}=1$, and $a_{n}=0$ for $n \neq 1$. The operator $H$ is real and is defined by

$$
H=\boldsymbol{\nabla} \cdot D \boldsymbol{\nabla} .
$$

In this section we show how the Green's function of the diffusion equation can be extracted from the correlation of fields excited by random sources. This derivation is equivalent to an earlier derivation [54].

For operator $H$ of expression (23), $f H g=f \nabla \cdot(D \nabla g)$ $=\boldsymbol{\nabla} \cdot(D f \nabla g)-D \boldsymbol{\nabla} \cdot f \boldsymbol{\nabla} g$. Integrating this over the volume $V$, applying Gauss's theorem, and subtracting the same expression with $f$ and $g$ interchanged, gives Green's theorem

$$
\int_{V}[f H g-(H f) g] d V=\oint_{\partial V} D\left(f \frac{\partial g}{\partial n}-\frac{\partial f}{\partial n} g\right) d S,
$$

where $\partial / \partial n$ denotes the derivative normal to the boundary $\partial V$. The bilinear form $L$ for this problem is thus given by

$$
L(f, g)=D\left(f \frac{\partial g}{\partial n}-\frac{\partial f}{\partial n} g\right) .
$$

Consider the special case, where on the boundary, either the field, its normal derivative, or a superposition of these quantities vanishes. This means that $f$ satisfies one of the following boundary conditions:

$$
f=0 \quad \text { or } \quad \frac{\partial f}{\partial n}=0 \quad \text { or } \quad \frac{\partial f}{\partial n}+\alpha f=0,
$$

with $\alpha$ a real number. The same boundary condition holds for $g$. In this case

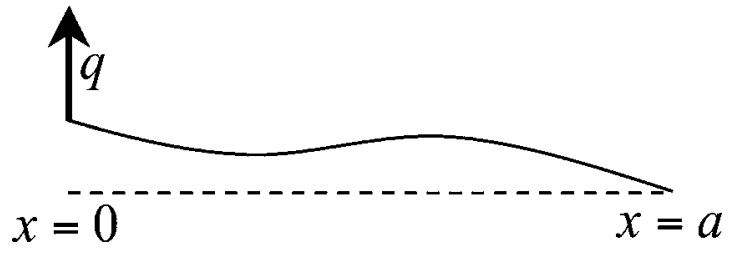

FIG. 3. A string with an open, forced, end at $x=0$ and a fixed end at $x=a$.

$$
L(f, g)=0,
$$

and expression (16), for the diffusion equation, is given by

$$
G\left(\mathbf{r}_{A}, \mathbf{r}_{B}\right)-G^{*}\left(\mathbf{r}_{A}, \mathbf{r}_{B}\right)=2 i \omega \int_{V} G\left(\mathbf{r}_{A}, \mathbf{r}\right) G^{*}\left(\mathbf{r}_{B}, \mathbf{r}\right) d V .
$$

Also, using expression (27), representation theorem (13) reduces to

$$
u\left(\mathbf{r}_{0}\right)=\int_{V} G\left(\mathbf{r}_{0}, \mathbf{r}\right) q(\mathbf{r}) d V .
$$

Suppose that the field is excited by spatially uncorrelated sources that have a power spectrum $|S(\omega)|^{2}$ :

$$
\left\langle q\left(\mathbf{r}_{1}\right) q^{*}\left(\mathbf{r}_{2}\right)\right\rangle=|S(\omega)|^{2} \delta\left(\mathbf{r}_{1}-\mathbf{r}_{2}\right) .
$$

The brackets $\langle\cdots\rangle$ denote an average of all sources. Expression (30) states that the excitation at two different spatial locations is uncorrelated when averaged over all sources. This happens for quasirandom continuous sources whose source signature is uncorrelated for sources at different locations. Equation (30) is also applicable when controlled, impulsive, sources fire sequentially at different locations, and when a summation over these sources is applied (e.g., Refs. $[24,25])$. In practical applications the source average for continuous sources is implemented by averaging over multiple nonoverlapping time windows, e.g., Refs. [20,36]. Multiplying expression (28) with $|S(\omega)|^{2}$, and using that

$$
\begin{aligned}
& \int_{V} G\left(\mathbf{r}_{A}, \mathbf{r}\right) G^{*}\left(\mathbf{r}_{B}, \mathbf{r}\right) d V \\
& \quad=\int_{V} \int_{V} G\left(\mathbf{r}_{A}, \mathbf{r}_{1}\right) \delta\left(\mathbf{r}_{1}-\mathbf{r}_{2}\right) G^{*}\left(\mathbf{r}_{B}, \mathbf{r}_{2}\right) d V_{1} d V_{2}
\end{aligned}
$$

gives

$$
\begin{aligned}
{\left[G\left(\mathbf{r}_{A}, \mathbf{r}_{B}\right)-G^{*}\left(\mathbf{r}_{A}, \mathbf{r}_{B}\right)\right]|S(\omega)|^{2} } & =2 i \omega \int_{V} \int_{V} G\left(\mathbf{r}_{A}, \mathbf{r}_{1}\right)|S(\omega)|^{2} \delta\left(\mathbf{r}_{1}-\mathbf{r}_{2}\right) G^{*}\left(\mathbf{r}_{B}, \mathbf{r}_{2}\right) d V_{1} d V_{2} \\
& =2 i \omega\left\langle\int_{V} G\left(\mathbf{r}_{A}, \mathbf{r}_{1}\right) q\left(\mathbf{r}_{1}\right) d V_{1}\left[\int_{V} G\left(\mathbf{r}_{B}, \mathbf{r}_{2}\right) q\left(\mathbf{r}_{2}\right) d V_{2}\right]^{*}\right\rangle=2 i \omega\left\langle u\left(\mathbf{r}_{A}\right) u^{*}\left(\mathbf{r}_{B}\right)\right\rangle,
\end{aligned}
$$


where expression (29) is used in the last identity. This result can be written as

$$
G\left(\mathbf{r}_{A}, \mathbf{r}_{B}\right)-G^{*}\left(\mathbf{r}_{A}, \mathbf{r}_{B}\right)=\frac{2 i \omega}{|S(\omega)|^{2}}\left\langle u\left(\mathbf{r}_{A}\right) u^{*}\left(\mathbf{r}_{B}\right)\right\rangle .
$$

The difference of the causal and acausal Green's function thus follows from the cross-correlation of fields excited by spatially uncorrelated sources. The factor $i \omega$ corresponds, in the time domain, to a (negative) time derivative $-\partial / \partial t$. A stronger excitation leads to stronger field, but the Green's functions in the left-hand side must be independent of the strength of the excitation of the fields that are correlated. The division by the power spectrum in the right hand side of expression (32) provides the required normalization.

The reason why volume sources are needed for the extraction of the Green's function can be explained as follows. The diffusion equation is of a dissipative nature. A continuous injection of energy within a volume is needed to overcome the dissipation inherent with diffusive systems. In this way an energy balance is established, and the system is equipartitioned when averaged over all sources.

\section{EXAMPLE: A STRING WITH MOVING END}

In order to clarify the essential role of an energy balance for retrieving the Green's function, we first present a simple one-dimensional system. Consider a string extending from $x=0$ to $x=a$ with mass-density $\rho(x)$ per unit length, and is under constant tension $T$, see Fig. 3. The left end of the string is excited at $x=0$, while the right end is fixed at $x=a$. There is no dissipation in the string. The motion of the string is governed by

$$
\rho(x) \frac{\partial^{2} u}{\partial t^{2}}-T \frac{\partial^{2} u}{\partial x^{2}}=q(x, t) .
$$

The string has a fixed end at $x=a$ and is being shaken by a force $F(t)$ at $x=0$

$$
q(x, t)=F(t) \delta(x) .
$$

In the notation of expression (1), $a_{2}(x)=\rho(x)$, all other $a_{n}$ are equal to zero, and $H=T \partial^{2} / \partial x^{2}$. Using definition (9)

$$
L(f, g)=T\left(f \partial_{x} g-g \partial_{x} f\right) .
$$

Inserting these results into expression (16) gives

$$
\begin{aligned}
G\left(x_{A}, x_{B}\right)-G^{*}\left(x_{A}, x_{B}\right)= & T\left[G^{*}\left(x_{B}, x\right) \partial_{x} G\left(x_{A}, x\right)\right. \\
& \left.-G\left(x_{A}, x\right) \partial_{x} G^{*}\left(x_{B}, x\right)\right]_{x=0}^{x=a} .
\end{aligned}
$$

[For one-dimensional systems the surface integral in expression (16) reduces to the difference of the integrand at the ends of the integration interval.] The contribution from the point $x=a$ on the right-hand side vanishes because the string is fixed at this point. In order to use this expression for the extraction of the Green's function, we need to eliminate the derivative of the Green's function at the left endpoint $(x$ $=0$ ) from this expression. This can be achieved by imposing a radiation boundary condition at the left side of the string. Together with the condition that the right side is fixed, this

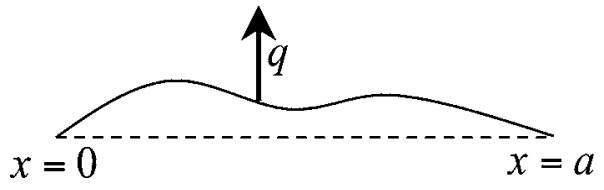

FIG. 4. A string that is fixed at both endpoints that is forced internally.

means that $u(x)$ and $G\left(x, x_{0}\right)$ satisfy the following boundary conditions

$$
\frac{\partial f(x=0)}{\partial x}=-i k f(x=0) \quad \text { and } \quad f(x=a)=0,
$$

with the local wave number $k$ at the left side of the string given by

$$
k=\frac{\omega}{c(x=0)},
$$

where $c=\sqrt{T / \rho}$. Note that for radiation boundary condition (37) the parameter $\alpha$ in expression (26) is imaginary. Inserting these results in expression (36) then gives

$$
G\left(x_{A}, x_{B}\right)-G^{*}\left(x_{A}, x_{B}\right)=2 i T k G\left(x_{A}, x=0\right) G^{*}\left(x_{B}, x=0\right) .
$$

Using boundary condition (37) the motion of string is according to expression (13) given by

$$
u\left(x_{0}\right)=G\left(x_{0}, x=0\right) F(\omega) .
$$

Multiplying Eq. (39) with $|F(\omega)|^{2}$, and using expression (40), then gives

$$
\begin{aligned}
{\left[G\left(x_{A}, x_{B}\right)-G^{*}\left(x_{A}, x_{B}\right)\right]|F(\omega)|^{2}=} & 2 i \operatorname{Tk} G\left(x_{A}, x=0\right) F(\omega) \\
& \times\left[G\left(x_{B}, x=0\right) F(\omega)\right]^{*} \\
= & 2 i \operatorname{Tku}\left(x_{A}\right) u^{*}\left(x_{B}\right) .
\end{aligned}
$$

Using expression (38), this result can also be written as

$$
G\left(x_{A}, x_{B}\right)-G^{*}\left(x_{A}, x_{B}\right)=\frac{2 i \omega \sqrt{\rho(x=0) T}}{|F(\omega)|^{2}} u\left(x_{A}\right) u^{*}\left(x_{B}\right) .
$$

In expression (41), the cross-correlation is multiplied with $T k$. The power in a vibrating string is proportional to $T k|u|^{2}$ [55]. This means that the reconstructed Green's function depends on the power that is injected into the string by the shaking at its end point.

We used the radiation boundary condition (37) to eliminate the $x$ derivative of the Green's function. The physical reason for this choice is that the radiation boundary condition corresponds to an energy sink by outward radiation at the same point where energy is supplied to the system (the left side of the string that is shaken). This creates a state of equipartitioning in the string. 


\section{EXAMPLE: A STRING WITH FIXED ENDPOINTS AND DISSIPATION}

In the previous example the string was not damped, and the radiation from the left end provided an energy sink. In this section we show a damped string with fixed endpoints, where the damping acts as an energy sink (see Fig. 4). The damped string with fixed endpoints satisfies

$$
\rho(x) \frac{\partial^{2} u}{\partial t^{2}}+a_{1}(x) \frac{\partial u}{\partial t}-T \frac{\partial^{2} u}{\partial x^{2}}=q(x, t),
$$

where $a_{1}(x)$ is the damping parameter. Because of the fixed endpoints

$$
u(x=0)=u(x=a)=0 .
$$

According to expression (35), for these boundary conditions the bilinear boundary term vanishes

$$
L(f, g)=0,
$$

and expression (16) is given by

$$
G\left(x_{A}, x_{B}\right)-G^{*}\left(x_{A}, x_{B}\right)=2 i \omega \int_{0}^{a} a_{1}(x) G\left(x_{A}, x\right) G^{*}\left(x_{B}, x\right) d x .
$$

For a given loading, the response according to Eq. (13) is given by

$$
u\left(x_{0}\right)=\int_{0}^{a} G\left(x_{0}, x\right) q(x) d x .
$$

Next consider a spatially uncorrelated excitation that satisfies

$$
\left\langle q\left(x_{1}\right) q^{*}\left(x_{2}\right)\right\rangle=a_{1}\left(x_{1}\right)|S(\omega)|^{2} \delta\left(x_{1}-x_{2}\right) .
$$

Note that this source strength locally is proportional to the attenuation, as described by the damping parameter $a_{1}(x)$. Multiplying expression (46) with the power spectrum gives

$$
\begin{aligned}
{\left[G\left(x_{A}, x_{B}\right)-G^{*}\left(x_{A}, x_{B}\right)\right]|S(\omega)|^{2} } & =2 i \omega \int_{0}^{a} \int_{0}^{a} G\left(x_{A}, x_{1}\right)|S(\omega)|^{2} a_{1}\left(x_{1}\right) \delta\left(x_{1}-x_{2}\right) G^{*}\left(x_{B}, x_{2}\right) d x_{1} d x_{2} \\
& =2 i \omega\left\langle\int_{0}^{a} G\left(x_{A}, x_{1}\right) q\left(x_{1}\right) d x_{1}\left(\int_{0}^{a} G\left(x_{B}, x_{2}\right) q\left(x_{2}\right) d x_{2}\right)^{*}\right\rangle=2 i \omega\left\langle u\left(x_{A}\right) u^{*}\left(x_{B}\right)\right\rangle
\end{aligned}
$$

hence the difference of the causal and acausal Green's functions follows from cross-correlation of the fields excited by spatially uncorrelated sources.

Note the presence of the damping $a_{1}(x)$ in expression (48). The Green's function can be extracted from the crosscorrelation only when the excitation is locally proportional to the damping. This creates an energy balance because the source of energy by the excitation is locally compensated by the attenuation, which acts as an energy sink. In Sec. XV we use the equation of radiative transfer to show that in a state of equipartitioning the excitation locally balances the dissipation due to intrinsic attenuation. When the excitation would not be proportional to the damping, there would be a net energy flux from the regions with strong excitation and weak damping to the areas of weak excitation and strong damping. The associated net energy flux violates the requirement of equipartitioning.

\section{EXAMPLE: FAILURE TO EXTRACT THE GREEN'S FUNCTION}

In this work numerous examples are presented of the extraction of the Green's function by cross-correlation. We now use the string, as presented in Secs. VII and VIII, to illustrate situations where the Green's function cannot be extracted by cross-correlation. First consider the string with internal exci- tation, as analyzed in Sec. VIII, but now without dissipation. This corresponds to the case $a_{1}=0$. Inserting this value in the right-hand side of expression (46) gives $G\left(x_{A}, x_{B}\right)$ $-G^{*}\left(x_{A}, x_{B}\right)=0$, which implies that the Green's function cannot be retrieved by cross-correlation.

The physical reason for the inability to extract the Green's function in this case is that energy is continuously supplied by the excitation, but there is no dissipation to act as a sink for this energy. The string thus is not in equilibrium, violating equipartitioning. Consequently, the Green's function cannot be retrieved from the fluctuations. In this case attenuation is needed to break the invariance for time-reversal in order to retrieve the Green's function.

As a second example consider the string that is excited at one of its endpoints, and whose motion is dissipative as described by a nonzero value for $a_{1}(x)$. Following expression (16), and modifying Eq. (39) to include the attenuation gives

$$
\begin{aligned}
G\left(x_{A}, x_{B}\right)-G^{*}\left(x_{A}, x_{B}\right)= & 2 i T k G\left(x_{A}, x=0\right) G^{*}\left(x_{B}, x=0\right) \\
& +2 i \omega \int_{0}^{a} a_{1}(x) G\left(x_{A}, x\right) G^{*}\left(x_{B}, x\right) d x
\end{aligned}
$$

Extending the steps leading to expression (41) then gives 


$$
\begin{aligned}
& {\left[G\left(x_{A}, x_{B}\right)-G^{*}\left(x_{A}, x_{B}\right)\right]|F(\omega)|^{2}} \\
& \quad=2 i T k u\left(x_{A}\right) u^{*}\left(x_{B}\right) \\
& \quad+2 i \omega|F(\omega)|^{2} \int_{0}^{a} a_{1}(x) G\left(x_{A}, x\right) G^{*}\left(x_{B}, x\right) d x .
\end{aligned}
$$

The last term prevents the Green's function from being retrieved by cross-correlation. Again, this is caused by a nonequilibrium state of this string. The string is being supplied with energy on its left endpoint while energy is being dissipated throughout the string. There is thus a net energy flux from the endpoint into the string. This net energy flux violates equipartitioning.

If in addition to the force at the endpoint, there also is a continuous excitation within the string, then the Green's function can be retrieved by cross-correlation. This can be achieved, however, only when the internal excitation compensates for the dissipation. Experimentally this may be difficult to realize.

\section{EXAMPLE: THE ACOUSTIC WAVE EQUATION}

The previous examples are for one-dimensional systems. The same principles hold for more dimensions. We show this by analyzing the acoustic wave equation (19) in more detail. This derivation is equivalent to earlier treatments [6,7]. A comparison with expression (23) shows that $H=\boldsymbol{\nabla} \cdot \rho^{-1} \boldsymbol{\nabla}$ is the same operator as that for the diffusion equation when $D$ is replaced by $\rho^{-1}$. Making this substitution in Eq. (25) and inserting these results in Eq. (16) gives

$$
\begin{aligned}
G\left(\mathbf{r}_{A}, \mathbf{r}_{B}\right)-G^{*}\left(\mathbf{r}_{A}, \mathbf{r}_{B}\right)= & \oint_{\partial V} \frac{1}{\rho}\left(G^{*}\left(\mathbf{r}_{B}, \mathbf{r}\right) \frac{\partial G\left(\mathbf{r}_{A}, \mathbf{r}\right)}{\partial n}\right. \\
& \left.-\frac{\partial G^{*}\left(\mathbf{r}_{B}, \mathbf{r}\right)}{\partial n} G\left(\mathbf{r}_{A}, \mathbf{r}\right)\right) d S .
\end{aligned}
$$

Note that in contrast to Eq. (28) for the diffusion equation, this expression contains a surface integral rather than a volume integral. Following Ref. [6] we use a spherical surface far away from the points $\mathbf{r}_{A}$ and $\mathbf{r}_{B}$ and impose a radiation boundary condition

$$
\frac{\partial G\left(\mathbf{r}_{0}, \mathbf{r}\right)}{\partial n}=i k G\left(\mathbf{r}_{0}, \mathbf{r}\right)
$$

Using the relation $k=\omega / c$, expression (52) is then given by

$$
G\left(\mathbf{r}_{A}, \mathbf{r}_{B}\right)-G^{*}\left(\mathbf{r}_{A}, \mathbf{r}_{B}\right)=2 i \omega \oint_{\partial V} \frac{1}{\rho c} G\left(\mathbf{r}_{A}, \mathbf{r}\right) G^{*}\left(\mathbf{r}_{B}, \mathbf{r}\right) d S .
$$

For spatially uncorrelated sources at the boundary that satisfy

$$
\left\langle q\left(\mathbf{r}_{1}\right) q^{*}\left(\mathbf{r}_{2}\right)\right\rangle=\frac{|S(\omega)|^{2}}{\rho\left(\mathbf{r}_{1}\right) c\left(\mathbf{r}_{1}\right)} \delta\left(\mathbf{r}_{1}-\mathbf{r}_{2}\right),
$$

expression (54) reduces to

$$
G\left(\mathbf{r}_{A}, \mathbf{r}_{B}\right)-G^{*}\left(\mathbf{r}_{A}, \mathbf{r}_{B}\right)=\frac{2 i \omega}{|S(\omega)|^{2}}\left\langle u\left(\mathbf{r}_{A}\right) u^{*}\left(\mathbf{r}_{B}\right)\right\rangle .
$$

In this case, energy is supplied to the system at the boundary by the sources, and the energy loss due to the radiation boundary condition establishes the equilibrium condition required for equipartitioning.

The presence of the factors $1 / \rho c$ in expression (55) can be explained as follows. The power in an acoustic medium is proportional to $p v$, with $v$ the particle velocity. The ratio of the pressure to the velocity is given by the acoustic impedance [56], $p / v=\rho c$; hence the power is proportional to $p^{2} / \rho c$. The excitation of expression (55) thus dictates that the power supplied at all points on the surface is constant, this establishes equipartitioning.

According to expression (56) the Green's function can indeed be extracted by cross-correlation. A similar problem has been formulated for acoustic waves that are attenuated [57], as described by a complex-valued compressibility $\kappa$ $=1 / \rho c^{2}$. In this case $\operatorname{Im}(H) \neq 0$, and, as a result, an additional volume integral is present in the right-hand side of expression (52). In that case the Green's function can be recovered when the volume is chosen in such a way that either the surface integral vanishes (i.e., a free surface) and volume sources are present, or the volume sources and the surface sources are in the right proportion as in the case of the string in Sec. IX.

\section{EXAMPLE: SCHRÖDINGER'S EQUATION}

The extraction of the Green's function can also be carried out for quantum systems. The extraction of the Green's function for Schrödinger's equation is almost the same as that for acoustic waves. (In quantum mechanics, the term "propagator" is often used for the Green's function [53].) For Schrödinger's equation (20), $H=-\left(\hbar^{2} / 2 m\right) \nabla^{2}+V$. A comparison with the acoustic wave equation (19) shows that in expression (52), $1 / \rho$ must be replaced by $-\hbar^{2} / 2 m$. Expression (54) generalizes for Schrödinger's equation to

$$
G\left(\mathbf{r}_{A}, \mathbf{r}_{B}\right)-G^{*}\left(\mathbf{r}_{A}, \mathbf{r}_{B}\right)=-\frac{i k \hbar^{2}}{m} \oint_{\partial V} G\left(\mathbf{r}_{A}, \mathbf{r}\right) G^{*}\left(\mathbf{r}_{B}, \mathbf{r}\right) d S .
$$

Suppose that the excitation on $\partial V$ is spatially uncorrelated, and is given by

$$
\left\langle q\left(\mathbf{r}_{1}\right) q^{*}\left(\mathbf{r}_{2}\right)\right\rangle=|S(\omega)|^{2} \delta\left(\mathbf{r}_{1}-\mathbf{r}_{2}\right) .
$$

Taking the same steps as in the derivation of expression (56), and denoting the field by $\psi$, the Green's function for Schrödinger's equation can be retrieved by cross-correlation

$$
G\left(\mathbf{r}_{A}, \mathbf{r}_{B}\right)-G^{*}\left(\mathbf{r}_{A}, \mathbf{r}_{B}\right)=-\frac{i k \hbar^{2}}{m|S(\omega)|^{2}}\left\langle\psi\left(\mathbf{r}_{A}\right) \psi^{*}\left(\mathbf{r}_{B}\right)\right\rangle .
$$

Extraction of the Green's function for Schrödinger's equation experimentally requires that the cross-correlation of the wave function be measured experimentally and that waves are excited at the bounding surface. The condition that 


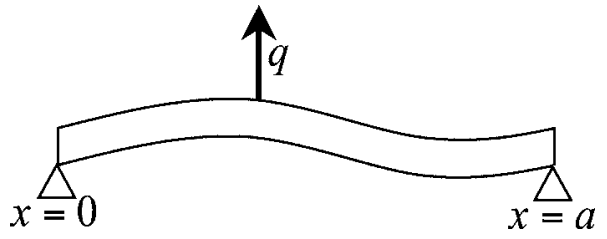

FIG. 5. A beam that is supported at both endpoints that is forced internally.

sources are present on the surface can be relaxed by using the derivation of Weaver and Lobkis [7], which shows for acoustic waves in a medium that is homogeneous outside the surface $\partial V$, that the sources on the surface can be replaced by distributed volume sources outside the surface. In the frequency domain, the acoustic wave equation for a homogeneous medium and Schrödinger's equation for a free particle both reduce to the Helmholtz equation. The arguments of Weaver and Lobkis therefore also are applicable for Schrödinger's equation when the potential vanishes outside the surface $\partial V$. The continuous source distribution is more realistic than sources on the surface for quantum-mechanical scattering problems.

The right-hand side of expression (59) contains the correlation of the fields at locations $\mathbf{r}_{A}$ and $\mathbf{r}_{B}$. This quantity can be measured when these points coincide $\mathbf{r}_{A}=\mathbf{r}_{B}=\mathbf{r}$. In that case

$$
G(\mathbf{r}, \mathbf{r})-G^{*}(\mathbf{r}, \mathbf{r})=-\frac{i k \hbar^{2}}{m|S(\omega)|^{2}}\left\langle|\psi(\mathbf{r})|^{2}\right\rangle .
$$

The right-hand side is the expectation value of the intensity fluctuations as a function of frequency. The left-hand side is the sum of the causal and acausal Green's function for waves to return to their starting point. This quantity contains phase information that is related to the time needed for a wave to return to the point $\mathbf{r}$ after it has left this point. The counterpart of expression (60) for elastic waves has been applied to recorded fluctuations in the displacement to determine the elastic waves that travel from a receiver into the subsurface of the Earth and then return to the receiver [36,37].

\section{EXAMPLE: A VIBRATING BEAM}

In the previous examples, the operator $H$ was a secondorder differential operator. This operator, however, need not be of second order. As an example, consider an unclamped beam that is supported at its end points $x=0$ and $x=a$; see Fig. 5. The beam satisfies a differential equation that is of fourth order in the space variable [58]

$$
m(x) \frac{\partial^{2} u}{\partial t^{2}}+a_{1}(x) \frac{\partial u}{\partial t}+\frac{\partial^{2}}{\partial x^{2}}\left(D(x) \frac{\partial^{2} u}{\partial x^{2}}\right)=q .
$$

In this expression $m(x)$ is the mass of the beam per unit length, and $D(x)$ denotes the flexural rigidity. In the notation of expression (1), $a_{2}(x)=m(x)$, and $H=-\partial_{x x}\left(D \partial_{x x}\right)$. Since the endpoints of the beam are fixed and unclamped, the beam satisfies the following boundary conditions:

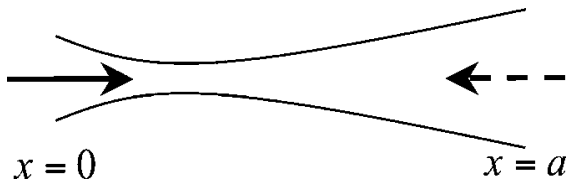

FIG. 6. Advective transport in a single direction.

$$
u(x=0)=u(x=a)=0 \text { and } \partial_{x x} u(x=0)=\partial_{x x} u(x=a)=0 .
$$

Using repeated integration by parts, the operator $L$ defined in expression (9) is given by

$$
L(f, g)=D\left(f_{x} g_{x x}-f_{x x} g_{x}\right)+g \partial_{x}\left(D f_{x x}\right)-f \partial_{x}\left(D g_{x x}\right) .
$$

Because of boundary conditions $(62),[L(f, g)]_{x=0}^{x=a}=0$. In this case, expression (16) is given by

$$
G\left(x_{A}, x_{B}\right)-G^{*}\left(x_{A}, x_{B}\right)=2 i \omega \int_{0}^{a} a_{1}(x) G\left(x_{A}, x\right) G^{*}\left(x_{B}, x\right) d x
$$

This expression is identical to Eq. (46) for the string with internal loading and attenuation. This means that for the beam the Green's function can be determined from the motion excited by the spatially uncorrelated source defined in Eq. (48) using the cross-correlation of expression (49). This example is of practical importance because the beam is a model for mechanical structures such as bridges.

\section{SYSTEMS DEFINED BY AN ANTISYMMETRIC OPERATOR}

The theory of the preceding sections is based on an operator $H$ that is symmetric when considered over the volume $V_{\text {tot }}$. Some systems are defined by an operator that is antisymmetric. This happens when $H$ contains an odd order of spatial derivatives, as in the flow problem presented in the next section. For such an operator

$$
\int_{V_{\mathrm{tot}}} f(H g) d V=-\int_{V_{\mathrm{tot}}}(H f) g d V
$$

Reciprocity does not hold in this case because the symmetry property of $H$ is essential in the derivation of expression (8). Equation (65) is not necessarily satisfied when the integration is carried out over a subvolume $V$. By analogy with expression (9) we define a bilinear form $M$ by

$$
\int_{V}[f(H g)+(H f) g] d V \equiv \oint_{\partial V} M(f, g) d S .
$$

A representation theorem of the correlation type follows by considering two states labeled with the subscripts $A$ and $B$, and by integrating the combination $(2)_{A} u_{B}^{*}+u_{A}(2)_{B}^{*}$ over the volume $V$ to give 


$$
\sum_{n=1}^{N} \int_{V}\left[(-i \omega)^{n} a_{n}+(i \omega)^{n} a_{n}^{*}\right] u_{A} u_{B}^{*} d V=\int_{V}\left[u_{B}^{*}\left(H u_{A}\right)+\left(H^{*} u_{B}^{*}\right) u_{A}\right] d V+\int_{V}\left(q_{A} u_{B}^{*}+q_{B}^{*} u_{A}\right) d V
$$

Applying steps similar to those for the derivation of expression (16) gives

$$
\begin{aligned}
G\left(\mathbf{r}_{B}, \mathbf{r}_{A}\right)+G^{*}\left(\mathbf{r}_{A}, \mathbf{r}_{B}\right)= & 2 \sum_{n \text { even }}(i \omega)^{n} \int_{V} \operatorname{Re}\left[a_{n}(\mathbf{r})\right] G\left(\mathbf{r}, \mathbf{r}_{A}\right) G^{*}\left(\mathbf{r}, \mathbf{r}_{B}\right) d V-2 i \sum_{n \text { odd }}(i \omega)^{n} \int_{V} \operatorname{Im}\left[a_{n}(\mathbf{r})\right] G\left(\mathbf{r}, \mathbf{r}_{A}\right) G^{*}\left(\mathbf{r}, \mathbf{r}_{B}\right) d V \\
& -\oint_{\partial V} M\left[G^{*}\left(\mathbf{r}, \mathbf{r}_{B}\right), G\left(\mathbf{r}, \mathbf{r}_{A}\right)\right] d S+2 i \int_{V} G\left(\mathbf{r}, \mathbf{r}_{A}\right) \operatorname{Im}(H) G^{*}\left(\mathbf{r}, \mathbf{r}_{B}\right) d V .
\end{aligned}
$$

Note that now for real $a_{n}$ only the even order coefficients contribute. Also, because of the lack of reciprocity, the arguments of the Green's function differ from those in the corresponding expression (16) for the case of a symmetric operator.

\section{EXAMPLE: THE ADVECTION EQUATION}

As a simple one-dimensional example of the extraction of the Green's function for a system that is described by an antisymmetric operator $H$, we study one-dimensional advection of a fluid. In this case the governing equation is

$$
\frac{1}{c(x)} \frac{\partial u}{\partial t}+\frac{\partial u}{\partial x}=q
$$

with the field $u$ advected by the flow. This field could denote, for example, the temperature for advective heat transport or the concentration of a nonreactive contaminant. Consider the case in which the field is determined by its value at a point upstream, rather than by an explicit source term; hence $q$ $=0$ in expression (69). As shown in Fig. 6, flow is between two endpoints $x=0$ and $x=a$, first with flow towards the right, as shown by the solid arrow. Because of the varying width of the channel, as in a venturi, the flow velocity may depend on the $x$ coordinate. For this system $a_{1}(x)=1 / c(x)$, all other $a_{n}$ are equal to zero, and $H=-\partial / \partial x$. Using integration by parts, the bilinear form $M$ defined in Eq. (66) is given by

$$
M(f, g)=-f g .
$$

We denote the Green's function which depends on the velocity $c$, by $G^{(c)}$. For this special case the general expression (68) reduces to

$$
G^{(c)}\left(x_{B}, x_{A}\right)+G^{(c)^{*}}\left(x_{A}, x_{B}\right)=\left[G^{(c)}\left(x, x_{A}\right) G^{(c) *}\left(x, x_{B}\right)\right]_{x=0}^{x=a} .
$$

Since $H$ is antisymmetric, reciprocity does not hold. For this type of flow problem, the flow-reversal theorem [59-61] states that the arguments of the Green's function can be reversed when the flow is reversed as well:

$$
G^{(c)}\left(x_{1}, x_{2}\right)=-G^{(-c)}\left(x_{2}, x_{1}\right) .
$$

Applying this to expression (71) gives

$$
G^{(-c)}\left(x_{A}, x_{B}\right)+G^{(-c)^{*}}\left(x_{B}, x_{A}\right)=-\left[G^{(-c)}\left(x_{A}, x\right) G^{(-c)^{*}}\left(x_{B}, x\right)\right]_{x=0}^{x=a} .
$$

Note that the velocity $c$ is replaced everywhere by $-c$. Since the sign of the velocity is not important, we drop the superscript $-c$, and consider a flow towards the left as shown by the dashed arrow in Fig. 6. For the advection equation a source has an influence downstream only; hence the Green's function for a leftward moving flow satisfies

$$
G\left(x, x_{0}\right)=0 \text { for } x>x_{0} .
$$

Consider two points between the endpoints of the flow $\left(0<x_{A, B}<a\right)$. Because of condition (74), the contribution of endpoint $x=0$ to expression (73) vanishes

$$
G\left(x_{A}, x_{B}\right)+G^{*}\left(x_{B}, x_{A}\right)=-G\left(x_{A}, x=a\right) G^{*}\left(x_{B}, x=a\right) .
$$

Let $u$ at the right endpoint have power spectrum $|S(\omega)|^{2}$. Multiplying expression (75) with this power spectrum, and taking the same steps as those leading to expression (41) gives

$$
G\left(x_{A}, x_{B}\right)+G^{*}\left(x_{B}, x_{A}\right)=\frac{-1}{|S(\omega)|^{2}} u\left(x_{A}\right) u^{*}\left(x_{B}\right) .
$$

This shows that the sum of the causal and acausal Green's functions can be found by correlating the field recorded at two locations that are generated by a source upstream.

For this problem, the sum of the causal and acausal Green's functions can be reformulated. Consider first the situation where $x_{B}$ is upstream from $x_{A}$; hence $x_{B}>x_{A}$. Because of condition (74), the second term in the left hand side of expression (76) vanishes, so that

$$
G\left(x_{A}, x_{B}\right)=\frac{-1}{|S(\omega)|^{2}} u\left(x_{A}\right) u^{*}\left(x_{B}\right)\left(x_{B}>x_{A}\right) .
$$

When $x_{B}$ is downstream from $x_{A}$, the first term of expression (76) vanishes by virtue of condition (74), and

$$
G^{*}\left(x_{B}, x_{A}\right)=\frac{-1}{|S(\omega)|^{2}} u\left(x_{A}\right) u^{*}\left(x_{B}\right)\left(x_{B}<x_{A}\right) .
$$

Taking the complex conjugate changes this result into 


$$
G\left(x_{B}, x_{A}\right)=\frac{-1}{|S(\omega)|^{2}} u\left(x_{B}\right) u^{*}\left(x_{A}\right)\left(x_{B}<x_{A}\right) .
$$

Expressions (77) and (79) can be combined into the general expression

$$
G\left(x_{\text {downstream }}, x_{\text {upstream }}\right)=\frac{-1}{|S(\omega)|^{2}} u\left(x_{\text {downstream }}\right) u^{*}\left(x_{\text {upstream }}\right),
$$

where $x_{\text {downstream }}$ denotes the downstream point and $x_{\text {upstream }}$ the upstream. This means that for the advection equation, the Green's function can be retrieved by cross-correlating the fields generated by a source upstream from both observation points. The extraction of the Green's function for acoustic waves in a medium with flow is described in Refs. [38,62,63]

\section{RECONSTRUCTING THE GREEN'S FUNCTION AND EQUIPARTITIONING}

As shown in the examples, an energy balance is necessary for extracting the Green's function by cross-correlation. This confirms the heuristic arguments of Sec. II. In this section we explore the requirement of equipartitioning. Let us first consider why the energy current, rather than another current such as the momentum current, plays such a central role in the extraction of the Green's function.

In the derivation of the general expression for extracting the Green's function in Sec. IV, a central step is to multiply the field equation for state $A$ with the complex conjugate of field for state $B$, and to integrate the result over volume, leading to expression (14). The field equation contains the forcing $q$. The product of the forcing and the field gives the power supplied by the excitation. This means that expression (14), and subsequent expression, really are energy equations. Fokkema, and van den Berg [50] use the phrase power reciprocity for the representation theorems of the correlation type.

For random media, a connection between the correlation and the energy transport is made through the Wigner distribution [64]. This distribution is the spatial Fourier transform of the field-field correlation function. Ryzhik et al. [65] show for acoustic waves, elastic waves, electromagnetic waves, and matter waves, that in random media the Wigner distribution leads to the equation of radiative transfer, which governs energy transport. Other derivations also used the Wigner distribution to show that for stationary random media, the Green's function of the mean field can be retrieved from the field correlations [42-44]. Larose et al. [2] discuss the relation between the Wigner distribution and the extraction of the Green's function in more detail.

We have stressed the importance of equipartitioning as defined by an energy current that is independent of direction. The energy current $J(\mathbf{r}, t, \hat{\mathbf{n}})$ satisfies the equation of radiative transfer, which, in the time domain, is given by [66-68]

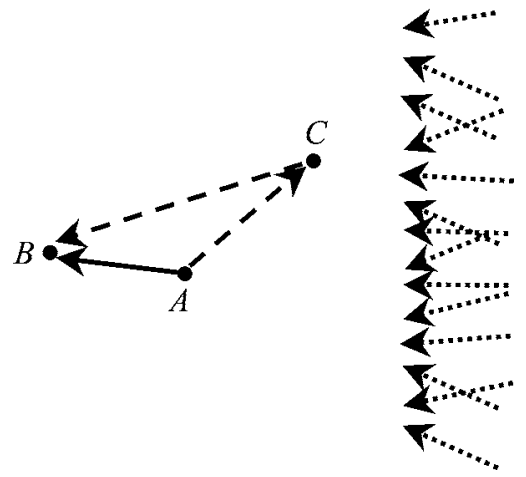

FIG. 7. An example in which the direct wave can be retrieved by cross correlation, but the diffracted wave cannot. Solid arrow: the direct wave propagating from $A$ to $B$. Dashed arrow: the diffracted wave traveling from $A$ through $C$ to $B$. Dotted arrows: direction of the incoming ambient waves used for the extraction of the Green's function.

$$
\begin{gathered}
\frac{\partial J(\mathbf{r}, t, \hat{\mathbf{n}})}{\partial t}+c \hat{\mathbf{n}} \cdot \nabla J(\mathbf{r}, t, \hat{\mathbf{n}})+\left(\mu_{\text {in }}+\mu_{\text {scat }}\right) J(\mathbf{r}, t, \hat{\mathbf{n}}) \\
=\int S(\hat{\mathbf{n}}, \hat{\mathbf{n}}) J(\mathbf{r}, t, \hat{\mathbf{n}}) d^{2} \hat{\mathbf{n}}+Q(\mathbf{r}, t, \hat{\mathbf{n}}) .
\end{gathered}
$$

In this expression $\mu_{\text {in }}$ and $\mu_{\text {scat }}$ are damping coefficients due to intrinsic attenuation and scattering losses, respectively. $S\left(\hat{\mathbf{n}}, \hat{\mathbf{n}}^{\prime}\right)$ accounts for the transfer of energy propagating in the $\hat{\mathbf{n}}^{\prime}$ direction to the $\hat{\mathbf{n}}$ direction by scattering, and $Q(\mathbf{r}, t, \hat{\mathbf{n}})$ denotes energy sources.

In our derivation of the extraction of the Green's function, we use source averaging. Since the source average does not depend on time, its time derivative vanishes. When the energy propagation is the same in all directions, the energy current does not depend on the direction of propagation $J$ $=J(\mathbf{r}, t)$. Consider the source-averaged intensity, which is defined as

$$
I(\mathbf{r})=\left\langle\int J(\mathbf{r}, t) d^{2} \hat{n}\right\rangle=4 \pi\langle J(\mathbf{r}, t)\rangle .
$$

When $J$ does not depend on the direction of propagation, the second term of the left-hand side of expression (81) integrates to zero; hence the average intensity satisfies in this case

$$
\left(\mu_{\text {in }}+\mu_{\text {scat }}\right) I(\mathbf{r})=\int S(\hat{\mathbf{n}}, \hat{\mathbf{n}}) d^{2} \hat{n} I(\mathbf{r})+\langle Q(\mathbf{r})\rangle,
$$

where $\langle Q\rangle$ is the source average of $Q$ averaged over all directions. The damping coefficient for scattering losses follows from the requirement that for lossless media $\left(\mu_{\text {in }}=0\right)$ in the absence of sources $(\langle Q\rangle=0)$, expression (83) reduces to

$$
\mu_{\text {scat }}=\int S(\hat{\mathbf{n}}, \hat{\mathbf{n}}) d^{2} \hat{n} .
$$

This expression relates the scattering attenuation to $S\left(\hat{\mathbf{n}}, \hat{\mathbf{n}}^{\prime}\right)$. This relation also holds in the presence of attenuation. Using the previous expression in Eq. (83) gives 


$$
\mu_{\text {in }} I(\mathbf{r})=\langle Q(\mathbf{r})\rangle .
$$

This means that for the source average in a equipartitioned state, the intrinsic attenuation is balanced by the energy sources. This is precisely the requirement that is obtained in Secs. VIII and XII for the damped string with fixed ends and for the damped vibrating beam. This condition is also required for attenuating acoustic waves [57].

\section{DISCUSSION}

The theory presented here shows that for a general class of scalar linear systems, the Green's function can be extracted from field correlations. This makes it possible to extract the Green's function for systems other than those for acoustic or elastic waves. Of particular interest are Schrödinger's equation and the diffusion equation, because the theory accounts for the extraction of the Green's function by cross-correlation in quantum mechanics, for the pore fluid pressure in porous media, the diffusive transport of tracers and contaminants, and for electromagnetic waves in attenuating media. The example of the vibrating beam has applications in monitoring bridges, buildings, and other mechanical structures.

The examples shown illustrate the importance of equipartitioning. This condition implies, in particular, that in systems that are not invariant under time-reversal, the sources of the field must be distributed throughout the volume and have a strength proportional to the local attenuation rate. Depending on the application, it might be difficult to realize such a distribution of sources experimentally.

It is not clear what happens when the requirement of equipartitioning is not satisfied. Figure 1 helps understand what happens in that case. Suppose the energy transport along the solid arrow is larger than the energy transport along the dashed arrow. The correlation of the field at the points $A$ and $B$ is larger than the correlation of the fields at the points $A$ and $C$. The extracted Green's function for the propagation from $A$ to $B$ is therefore stronger than those from $A$ to $C$. The arrival time, or phase, of the Green's function, however, is not influenced by this mismatch in the energy flow. This suggests that when the condition of equipartitioning is violated, the kinematic properties of the extracted Green's function is correct, although the dynamic properties are not. Experiments with ultrasound [18] and crustal surface waves [49] support this conclusion. This conclusion is also supported by analytic models which show that for elastic waves in a homogeneous medium, the amplitude of the $P$ and $S$ waves in the extracted Green's function is correct only when the ratio of the $P$-wave energy to the $S$-wave energy equals the value required by equipartitioning, but the phase of the extracted $P$ and $S$ waves is correct for any value of this ratio $[8,9]$.

Two caveats should be made about the need for equipartitioning. First, note that in the advection example of Sec. XIV there is no need to excite the field on the downstream side. All transport is in the flow direction only; hence equipartitioning is unnecessary in that example. Second, the formalism for the extraction of the Green's function given here gives the superposition of the causal and acausal Green's function. In some situations, such as a homogeneous medium, one-sided energy transport is sufficient to give either the causal or the acausal Green's function. This is illustrated in Fig. 7 showing incoherent waves are propagating towards the left (dotted arrows). Here, the direct wave propagating from $A$ to $B$, as indicated by the solid arrow, can be retrieved by cross-correlation, but the direct wave traveling in the opposite direction cannot. This does not mean, however, that the full Green's function for wave propagation from $A$ to $B$ can be retrieved. Suppose a diffractor is present at point $C$. The diffracted wave traveling from $A$ through $C$ to $B$, as shown by the dashed arrows, cannot be extracted from the waves coming in from the right. Cross correlation here gives only part of the Green's function (the direct wave).

The words equipartitioning and ensemble average have a well-defined meaning in statistical mechanics that does not necessarily carry over to the macroscopic systems considered here. Note that we have not assumed thermodynamic equilibrium, as used in derivations of the fluctuation-dissipation theorem [39-41]. In thermodynamic equilibrium, a state with energy $E$ is weighted by $\exp (-\beta E)$ in the ensemble average, where $\beta^{-1}=k T$ is the thermal energy. In this work, the fields are characterized by a power spectrum $|S(\omega)|^{2}$ that can be any function of frequency, as long as it is known. The fact that thermodynamic equilibrium is not required is no surprise because the field energy in the macroscopic systems considered here is usually much larger than the thermal energy.

This implies that source average in the context of this paper does not refer to a thermodynamic average. This means, in particular, that at any given moment in time, the system need not be close to a state of equilibrium. Consider, for example, a string that is excited at both endpoints. It does not matter whether the two endpoints are simultaneously excited with an uncorrelated forcing, or one first shakes one endpoint and then the other endpoint. In fact, in the virtual source method $[24,25]$ one excites elastic waves sequentially by different sources, and extracts the Green's function by summing over all the sources. Averaging in the context of this work implies an averaging over all sources that are used for the extraction of the Green's function, and equipartitioning is required after averaging over these sources.

\section{ACKNOWLEDGMENTS}

We appreciate critical and illuminating discussions with Ken Larner and Rodney Calvert.

\section{APPENDIX: AN EXAMPLE THAT A VANISHING NET ENERGY CURRENT DOES NOT IMPLY EQUIPARTITIONING}

The net energy transport $\mathbf{J}^{(\text {net })}$ is the energy current averaged over all directions

$$
\mathbf{J}^{(\mathrm{net})}=\oint J(\hat{\mathbf{n}}) \hat{\mathbf{n}} d^{2} n,
$$

where $\oint(\cdots) d^{2} n$ denotes an integration over all directions. At every point in space the energy current can be expanded in spherical harmonics 


$$
J(\theta, \varphi)=\sum_{l, m} J_{l, m} Y_{l, m}(\theta, \varphi)
$$

where the angles $\theta$ and $\varphi$ are the polar angles of the propagation direction $\hat{\mathbf{n}}$ :

$$
\hat{\mathbf{n}}=\left(\begin{array}{c}
\sin \theta \cos \varphi \\
\sin \theta \sin \varphi \\
\cos \theta
\end{array}\right) .
$$

Rather than considering the Cartesian components of $\hat{\mathbf{n}}$, we consider for brevity [69]

$$
n_{x} \pm i n_{y}=\sin \theta \exp ( \pm i \varphi)=\mp \sqrt{\frac{8 \pi}{3}} Y_{1, \pm 1}(\theta, \varphi)
$$

and

$$
n_{z}=\cos \theta=\sqrt{\frac{4 \pi}{3}} Y_{1,0}(\theta, \varphi)
$$

The corresponding components of the net energy current are given by

$$
J_{x}^{(\mathrm{net})} \pm i J_{y}^{(\mathrm{net})}=\sum_{l, m} J_{l, m} \oint Y_{l, m}(\theta, \varphi) \sin \theta e^{ \pm i \varphi} d^{2} n
$$

and

$$
J_{z}^{(\mathrm{net})}=\sum_{l, m} J_{l, m} \oint Y_{l, m}(\theta, \varphi) \cos \theta d^{2} n .
$$

Because of the orthogonality of the spherical harmonics [69]

$$
J_{x}^{(\mathrm{net})} \pm i J_{y}^{(\mathrm{net})}=\mp \sqrt{\frac{8 \pi}{3}} J_{1, \mp 1}, \quad J_{z}^{(\mathrm{net})}=\sqrt{\frac{4 \pi}{3}} J_{1,0} .
$$

The condition that the net energy current vanishes thus implies that

$$
J_{l=1, m}=0, \quad m=0, \pm 1 .
$$

A vanishing energy current thus requires only that the coefficients $J_{l=1, m}$ vanish. For a vanishing net energy current $\mathbf{J}^{\text {(net) }}=0$, all coefficients $J_{l, m}$ with $l \neq 1$ can be nonzero. This means that an energy current given by expansion (A2) with nonzero coefficients $J_{l, m}$ for $l>1$ gives a vanishing net energy current, while the energy current $J(\hat{\mathbf{n}})$ varies with direction. In this case the net energy current vanishes, but there is no equipartitioning.
[1] A. Curtis, P. Gerstoft, H. Sato, R. Snieder, and K. Wapenaar, The Leading Edge 25, 1082 (2006).

[2] E. Larose, L. Margerin, A. Derode, B. van Tiggelen, M. Campillo, N. Shapiro, A. Paul, L. Stehly, and M. Tanter, Geophysics 71, SI11 (2006).

[3] K. Wapenaar, D. Dragonv, and J. Robertsson, Geophysics 71, SI1 (2006).

[4] O. I. Lobkis and R. L. Weaver, J. Acoust. Soc. Am. 110, 3011 (2001).

[5] K. Wapenaar, Phys. Rev. Lett. 93, 254301 (2004).

[6] K. Wapenaar, J. Fokkema, and R. Snieder, J. Acoust. Soc. Am. 118, 2783 (2005).

[7] R. L. Weaver and O. I. Lobkis, J. Acoust. Soc. Am. 116, 2731 (2004).

[8] F. J. Sánchez-Sesma, J. A. Pérez-Ruiz, M. Campillo, and F. Luzón, Geophys. Res. Lett. 33, L13305 (2006).

[9] F. J. Sánchez-Sesma and M. Campillo, Bull. Seismol. Soc. Am. 96, 1182 (2006).

[10] A. Derode, E. Larose, M. Tanter, J. de Rosny, A. Tourin, M. Campillo, and M. Fink, J. Acoust. Soc. Am. 113, 2973 (2003).

[11] A. Derode, E. Larose, M. Campillo, and M. Fink, Appl. Phys. Lett. 83, 3054 (2003).

[12] R. Snieder, Phys. Rev. E 69, 046610 (2004).

[13] P. Roux, K. G. Sabra, W. A. Kuperman, and A. Roux, J. Acoust. Soc. Am. 117, 79 (2005).

[14] K. G. Sabra, P. Roux, and W. A. Kuperman, J. Acoust. Soc. Am. 117, 164 (2005).

[15] R. Snieder, K. Wapenaar, and K. Larner, Geophysics 71, SI111 (2006).

[16] R. L. Weaver and O. I. Lobkis, Phys. Rev. Lett. 87, 134301
(2001).

[17] R. Weaver and O. Lobkis, Ultrasonics 40, 435 (2003).

[18] A. E. Malcolm, J. A. Scales, and B. A. van Tiggelen, Phys. Rev. E 70, 015601(R) (2004).

[19] M. Campillo and A. Paul, Science 299, 547 (2003).

[20] N. M. Shapiro and M. Campillo, Geophys. Res. Lett. 31, L07614 (2004).

[21] N. M. Shapiro, M. Campillo, L. Stehly, and M. H. Ritzwoller, Science 307, 1615 (2005).

[22] K. G. Sabra, P. Gerstoft, P. Roux, W. A. Kuperman, and M. C. Fehler, Geophys. Res. Lett. 32, L14311 (2005).

[23] K. G. Sabra, P. Gerstoft, P. Roux, W. A. Kuperman, and M. C. Fehler, Geophys. Res. Lett. 32, L03310 (2005).

[24] A. Bakulin and R. Calvert, in Expanded Abstracts of the 2004 SEG Meeting (Society of Exploration Geophysicists, Tulsa, OK, 2004), pp. 2477-2480.

[25] A. Bakulin and R. Calvert, Geophysics 71, SI139 (2006).

[26] G. T. Schuster, J. Yu, J. Sheng, and J. Rickett, Geophys. J. Int. 157, 838 (2004).

[27] J. E. Rickett and J. F. Claerbout, The Leading Edge 18, 957 (1999).

[28] J. E. Rickett and J. F. Claerbout, Sol. Phys. 192, 203 (2000).

[29] J. E. Rickett and J. F. Claerbout, in Helioseismic Diagnostics of Solar Convection and Activity, edited by T. L. Duvall, J. W. Harvey, A. G. Kosovichev, and Z. Svestka (Kluwer, Dordrecht 2001).

[30] R. Snieder and E. Şafak, Bull. Seismol. Soc. Am. 96, 586 (2006).

[31] R. Snieder, J. Sheiman, and R. Calvert, Phys. Rev. E 73, 066620 (2006). 
[32] D. Thompson and R. Snieder, The Leading Edge 25, 1093 (2006).

[33] P. Roux, W. A. Kuperman, and NPAL Group, J. Acoust. Soc. Am. 116, 1995 (2004).

[34] K. G. Sabra, P. Roux, A. M. Thode, G. L. D’Spain, and W. S. Hodgkiss, IEEE J. Ocean. Eng. 30, 338 (2005).

[35] K. Mehta, R. Snieder, and V. Graizer, Geophys. J. Int. 168, (2007).

[36] C. Sens-Schönfelder and U. Wegler, Geophys. Res. Lett. 33, L21302 (2006).

[37] U. Wegler and C. Sens-Schönfelder, Geophys. J. Int. 168, 1029 (2007).

[38] K. Wapenaar, E. Slob, and R. Snieder, Phys. Rev. Lett. 97, 234301 (2006).

[39] H. B. Callen and T. A. Welton, Phys. Rev. 83, 34 (1951).

[40] R. Kubo, Rep. Prog. Phys. 29, 255 (1966).

[41] M. Le Bellac, F. Mortessagne, and G. G. Batrouni, Equilibrium and Non-equilibrium Statistical Thermodynamics (Cambridge Univesity Press, Cambridge, UK, 2004).

[42] Yu. N. Barabanenkov, Izv. Vyssh. Uchebn. Zaved., Radiofiz. 12, 894 (1969).

[43] Yu. N. Barabanenkov, Izv. Vyssh. Uchebn. Zaved., Radiofiz. 14, 887 (1971)

[44] S. M. Rytov, Yu. A. Kravtsov, and V. I. Tatarskii, Principles of Statistical Radiophysics (Springer, Berlin, 1989), Vol. 3.

[45] R. L. Weaver, J. Acoust. Soc. Am. 71, 1608 (1982).

[46] H. Goldstein, Classical Mechanics, 2nd ed. (Addison-Wesley, Reading, MA, 1980).

[47] C. Chapman, Fundamentals of Seismic Wave Propagation (Cambridge University Press, Cambridge, UK, 2004).

[48] K. Aki and P. G. Richards, Quantitative Seismology, 2nd ed. (University Science Books, Sausalito, 2002).

[49] A. Paul, M. Campillo, L. Margerin, E. Larose, and A. Derode, J. Geophys. Res. 110, B08302 (2005).

[50] J. T. Fokkema and P. M. van den Berg, Seismic Applications of Acoustic Reciprocity (Elsevier, Amsterdam 1993).
[51] J. T. Fokkema and P. M. van den Berg, in Wavefields and Reciprocity, edited by P. M. van den Berg, H. Blok, and J. T. Fokkema, (Delft University Press, Delft, 1996), pp. 99-108.

[52] R. L. Weaver and O. I. Lobkis, J. Acoust. Soc. Am. 117, 3432 (2005).

[53] E. Merzbacher, Quantum Mechanics, 2nd ed. (Wiley, New York 1970).

[54] R. Snieder, Phys. Rev. E 74, 046620 (2006).

[55] E. Butkov, Mathematical Physics (Addison-Wesley, Reading MA 1968).

[56] R. Snieder, A Guided Tour of Mathematical Methods for the Physical Sciences, 2nd ed. (Cambridge University Press, Cambridge, 2004).

[57] R. Snieder, J. Acoust. Soc. Am. (to be published).

[58] A. K. Chopra, Dynamics of Structures; Theory and Applications to Earthquake Engineering, 2nd ed. (Prentice Hall, New York, 1995).

[59] L. M. Lyamshev, Dokl. Akad. Nauk SSSR 138, 575 (1961).

[60] L. M. Brekhovskikh and O. A. Godin, Acoustics of Layered Media II. Point Sources and Boudary Beams (Springer Verlag, Berlin, 1992).

[61] K. Wapenaar and J. Fokkema, J. Appl. Mech. 71, 145 (2004).

[62] K. Wapenaar, J. Acoust. Soc. Am. 120, EL7 (2006).

[63] O. A. Godin, Phys. Rev. Lett. 97, 054301 (2006).

[64] E. Wigner, Phys. Rev. 40, 749 (1932).

[65] L. Ryzhik, G. Papanicolaou, and J. B. Keller, Wave Motion 24, 327 (1996).

[66] S. Chandrasekhar, Radiative Transfer (Dover, New York 1960).

[67] M. N. Özisik, Radiative Transfer and Interaction with Conduction and Convection (John Wiley, New York, 1973).

[68] S. M. Rytov, Yu. A. Kravtsov, and V. I. Tatarskii, Principles of Statistical Radiophysics (Springer, Berlin 1989), Vol. 4.

[69] G. B. Arfken and H. J. Weber, Mathematical Methods for Physicists, 5th ed. (Harcourt, Amsterdam, 2001). 